\title{
Resultados perinatales en fetos con flujo umbilical diastólico ausente
}

\author{
Yurena Méndez $L_{i}{ }^{1}$, Elsa Estévez $R{ }^{2}$, Margarita Álvarez De La Rosa $R{ }^{1}$, \\ Marta Coloma E. ${ }^{1}$, Ana Isabel Padilla $P^{1}{ }^{1}$, Juan Mario Troyano $L .^{1}$ \\ 1 Servicio de Ginecología y Obstetricia del Complejo Hospitalario Universitario de Tenerife. ${ }^{2}$ Servicio de Ginecología y \\ Obstetricia del Hospital General de La Palma. Islas Canarias, España.
}

\section{RESUMEN}

Objetivo: Estudiar los resultados perinatales de fetos diagnosticados con flujo umbilical ausente en diástole en el Hospital Universitario de Canarias, España. Métodos: Estudio retrospectivo de gestantes con Doppler fetal umbilical con flujo diastólico ausente entre 2004 y 2011, excluyendo embarazos gemelares. Las variables estudiadas fueron: edad y enfermedades maternas, edad gestacional al diagnóstico y parto, vía de parto, Apgar y estado del recién nacido. Resultados: Se recogieron 57 casos. Hubo un $43 \%$ de gestantes hipertensas y $19 \%$ de diabéticas. La edad gestacional media al diagnóstico fue de $30+4$ semanas. En el $89 \%$ de los casos se observó redistribución del flujo y en el $21 \%$ el Doppler umbilical fue reverso. El $28 \%$ se acompañó de oligoamnios y en el $26 \%$ el peso fetal estimado inferior al percentil 3 . Hubo 3 muertes fetales. En el $80 \%$ de los casos la vía de parto fue por cesárea. La supervivencia fue del $83 \%$ con una tasa de mortalidad perinatal del $17 \%$. En el seguimiento entre 1 y 6 años encontramos un $35 \%$ de niños sin secuelas. Conclusiones: En nuestra serie, el Doppler con flujo umbilical ausente en diástole se relaciona con alto riesgo de morbimortalidad perinatal. El $65 \%$ de los niños tendrán secuelas de algún tipo en el seguimiento entre 1 a 6 años. El momento óptimo para la extracción fetal debe ser individualizado y resuelto junto con el consejo pediátrico y participación de los padres.

\section{PALABRAS CLAVE: Doppler arteria umbilical, flujo diastólico ausente, morbilidad y mortalidad perinatal, restricción del crecimiento intrauterino}

\section{SUMMARY}

Objective: To determine the perinatal outcome in fetal growth restriction were umbilical artery Doppler end diastolic flow was absent. Methods: A retrospective study performed at the Canary Islands University Hospital. All consecutive cases between 2004 and 2011 were included. We excluded twin pregnancies. Data was abstracted for maternal age, gestational age at diagnosis and delivery, mode of delivery, Apgar and perinatal adverse outcomes. Results: Fifty seven patients were included. Forty three percent had a pregnancy complicated by hypertension and $19 \%$ diabetes. Mean gestational age at diagnosis was $30+4$ weeks. We found $89 \%$ and $21 \%$ absent umbilical artery end diastolic flow and reverse flow respectively. Oligohydramnios was seen in $28 \%$ of the cases. Fetal weight was below the third percentile in $26 \%$ of the cases. Fetal demise occurred in 3 cases. Mode of delivery was cesarean section in $80 \%$ of the cases. We found a rate of $17 \%$ perinatal mortality. At 1 to 6 years follow up 35\% of the children had no sequelae. Conclusion: Absent end diastolic umbilical flow is associated with a high risk of perinatal mortality. Up to $65 \%$ of the children will show some type of sequelae. The optimal gestational date for fetal extraction should be individualized after multidisciplinary counseling.

KEY WORDS: Umbilical artery Doppler, absent end diastolic umbilical flow, perinatal outcome, intrauterine growth restriction 


\section{INTRODUCCIÓN}

El flujo diastólico ausente en la arteria umbilical identifica a los fetos con crecimiento intrauterino retardado (CIR) con alto riesgo de morbimortalidad perinatal. La alteración de la vascularización placentaria desencadena a nivel de la circulación fetoplacentaria cambios hemodinámicos progresivos en relación a la adaptación fetal a la hipoxia. Se produce una redistribución del flujo sanguíneo mediante una vasoconstricción selectiva en determinados órganos mientras que se mantiene o aumenta el flujo en otros como cerebro, miocardio y glándulas suprarrenales $(1,2)$. Estos cambios conocidos como brain sparing pueden detectarse mediante las técnicas de ecografía e índices Doppler, siendo la secuencia esperada la siguiente: aumento de la resistencia en la arteria umbilical, disminución de resistencias en la arteria cerebral media, ausencia de diástole en la arteria umbilical, aparición de flujo reverso en la arteria umbilical, reducción de flujo o reversión del ductus venoso y vena cava inferior, pulsatilidad de la vena umbilical, que finalmente se correlaciona con acidosis fetal, con gran riesgo de alteración neurológica y muerte fetal $(2,3)$.

Estos cambios son predecibles en los fetos de menos de 34 semanas hasta en el $70 \%$ de los casos (4), y su estudio nos puede ayudar a la elección del momento más oportuno para la extracción fetal sobretodo en los casos de edades gestacionales extremas donde se debe intentar maximizar la edad gestacional para minimizar la morbimortalidad neonatal $(5,6)$.

El objetivo es analizar los resultados perinatales de los fetos con flujo diastólico ausente en arteria umbilical, atendidos en el Hospital Universitario de Canarias.

\section{PACIENTES Y MÉTODO}

Estudio de cohorte retrospectiva en el que se revisan las historias clínicas de 57 gestantes consecutivas con flujo diastólico ausente en arteria umbilical fetal, diagnosticadas en el Hospital Universitario de Canarias entre los años 2004-2011, y la evolución perinatal. Se excluyeron los embarazos múltiples. Las variables recogidas fueron: edad, paridad y antecedentes médicos maternos, edad gestacional en el momento del diagnóstico de Doppler umbilical con flujo ausente en diástole y al parto, número de días transcurridos desde el diagnóstico hasta la extracción fetal, administración de maduración pulmonar fetal, vía de parto, Apgar, peso del recién nacido y condición en la actualidad. Se empleó el programa SPSS (versión 17, IBM) para el análisis estadístico descriptivo de los resultados obtenidos. Todas las pacientes fueron diagnosticadas por la unidad de ecografía y diagnóstico prenatal de nuestro centro y tratadas según los protocolos del Servicio de Obstetricia, basados fundamentalmente en ingreso de la paciente, ecografía Doppler y perfil biofísico cada 24 horas, registro cardiotocográfico cada 12-24 horas según gravedad del caso y control de la patología de base de la paciente. El CIR fue definido como percentil de peso fetal estimado menor de 3 para la edad gestacional, utilizando para la determinación del peso fetal el método de Persson (diámetro biparital, perímetro abdominal y longitud de fémur) y curvas españolas de crecimiento de fetal para la determinación de percentiles (SEGO, disponible en www.fetaltest.com). El oligoamnios fue definido como columna máxima de líquido amniótico menor a 2 o índice de líquido amniótico inferior a 5. La centralización fue definida como índice de pulsatilidad en la arteria cerebral media menor del percentil 5, o índice cerebro placentario igual o inferior a 1.

Los resultados perinatales adversos fueron definidos como necesidad de apoyo respiratorio fetal, ya sea con métodos invasivos o no invasivos (distrés respiratorio, síndrome de membrana hialina, apnea primaria, displasia broncopulmonar, neumotórax, entre otros), necesidad de aminas vaso activas, hemorragia intraventricular, sepsis precoz o tardía, retinopatía del prematuro en cualquiera de sus grados, leucomalacia ventricular, enterocolitis necrotizante y necesidad de transfusión (anemia y/o trombopenia del prematuro). Las secuelas fueron definidas como necesidad de cirugía o rehabilitación por patología del prematuro, retraso psicomotor y/o del crecimiento, epilepsia y parálisis cerebral.

\section{RESULTADOS}

Se recogieron 57 casos consecutivos con edades maternas comprendidas entre los 19 y los 44 años (media: 30,8 años) siendo 41 primigestas (72\%). En la Tabla I se presentan los antecedentes personales y clínicos de las gestantes.

En las 16 pacientes multíparas (28\%) encontramos como antecedentes obstétricos 1 hemorragia puerperal tras parto normal, 4 pacientes con 2 ó más abortos espontáneos, 2 enfermedades hipertensivas gestacionales durante la gestación previa, 1 parto prematuro previo y dos pacientes con interrupción voluntaria del embarazo previo por patología fetal: una por CIR precoz y otra por mielomeningocele. La edad gestacional media al diagnóstico fue de 30,4 semanas (rango: 24,1 - 38,3 semanas).

En 51 casos (89\%) existía centralización y en $12(21 \%)$ el Doppler umbilical fue reverso. En 16 pacientes (28\%) el diagnóstico se acompañó de oligoamnios y en 15 casos (26\%) con peso fetal $<$ percentil 3. 
Tabla I

ANTECEDENTES PERSONALES Y CLÍNICOS MATERNOS

\begin{tabular}{ll}
\hline \multicolumn{1}{c}{ Variable } & \multicolumn{1}{c}{ Medidas } \\
\hline Edad materna & $\begin{array}{l}\text { Rango: } 19-44 \text { años } \\
\text { Media: 30,8 años }\end{array}$ \\
Paridad & $\begin{array}{l}\text { Primigestas } 41(72 \%) \\
\text { Multíparas 16 }(28 \%)\end{array}$ \\
Hipertensión arterial & $25(43 \%)$ \\
Diabetes & $11(19 \%)$ \\
Diabetes + hipertensión arterial & $5(8 \%)$ \\
Fumadoras & $6(10 \%)$ \\
Técnica de reproducción asistida & $6(10 \%)$ \\
Enfermedades reumatológicas & $1(2 \%)$ \\
Enfermedades infecciosas & $1(2 \%)$ \\
\hline
\end{tabular}

El promedio de tiempo desde el diagnóstico hasta el parto fue de 8 días (rango: 0-70 días), administrándose en 31 casos (54\%) maduración pulmonar fetal, parcial o completa (una o dos dosis de $12 \mathrm{mg}$ de betametasona intramuscular administradas en un intervalo de 24 horas). Se obtuvo un Apgar a los cinco minutos $<6$ en 4 casos y $\mathrm{pH}<7.20$ en 10 casos (Figura 1 y Tabla II).
Hubo 3 muertes fetales: 2 de ellos con Doppler reverso, y 7 muertes posnatales, todos menores de 30 semanas; 6 nacieron por cesárea. Tres tuvieron un Apgar $<6$ a los 5 minutos, 6 tenían centralización, todos ellos $\mathrm{CIR}$ y pesos al nacer $<900$ gramos (rango: $500-898 \mathrm{~g}$ ). La supervivencia fue del $83 \%$ con una tasa de mortalidad perinatal del $17 \%$ (Tabla III).

De los 54 casos de recién nacidos vivos se pudo obtener datos de 48 de ellos entre los que se incluyen 6 de las muertes posnatales. La estancia media hospitalaria de los recién nacidos fue de 37 días (rango:1-190 días). Los resultados adversos más frecuentes encontrados se resumen en la Tabla IV.

El $92 \%$ de los recién nacidos $<1500$ gramos tuvo algún tipo de complicación y el $100 \%$ de los $<1000$ gramos tuvo complicaciones graves por prematuridad.

\section{DISCUSIÓN}

Nuestros resultados confirman el alto riesgo de morbimortalidad perinatal en los casos de ausencia de flujo umbilical en diástole. En nuestra serie el Doppler con flujo umbilical ausente en diástole se relaciona con la enfermedad hipertensiva y diabetes materna, principalmente. Hubo un $17 \%$ de mortalidad perinatal y aproximadamente el $65 \%$ de los niños tuvo secuelas de algún tipo. La vía del parto fue la cesárea en el $50 \%$ de los intentos de inducción y globalmente en el $80 \%$.

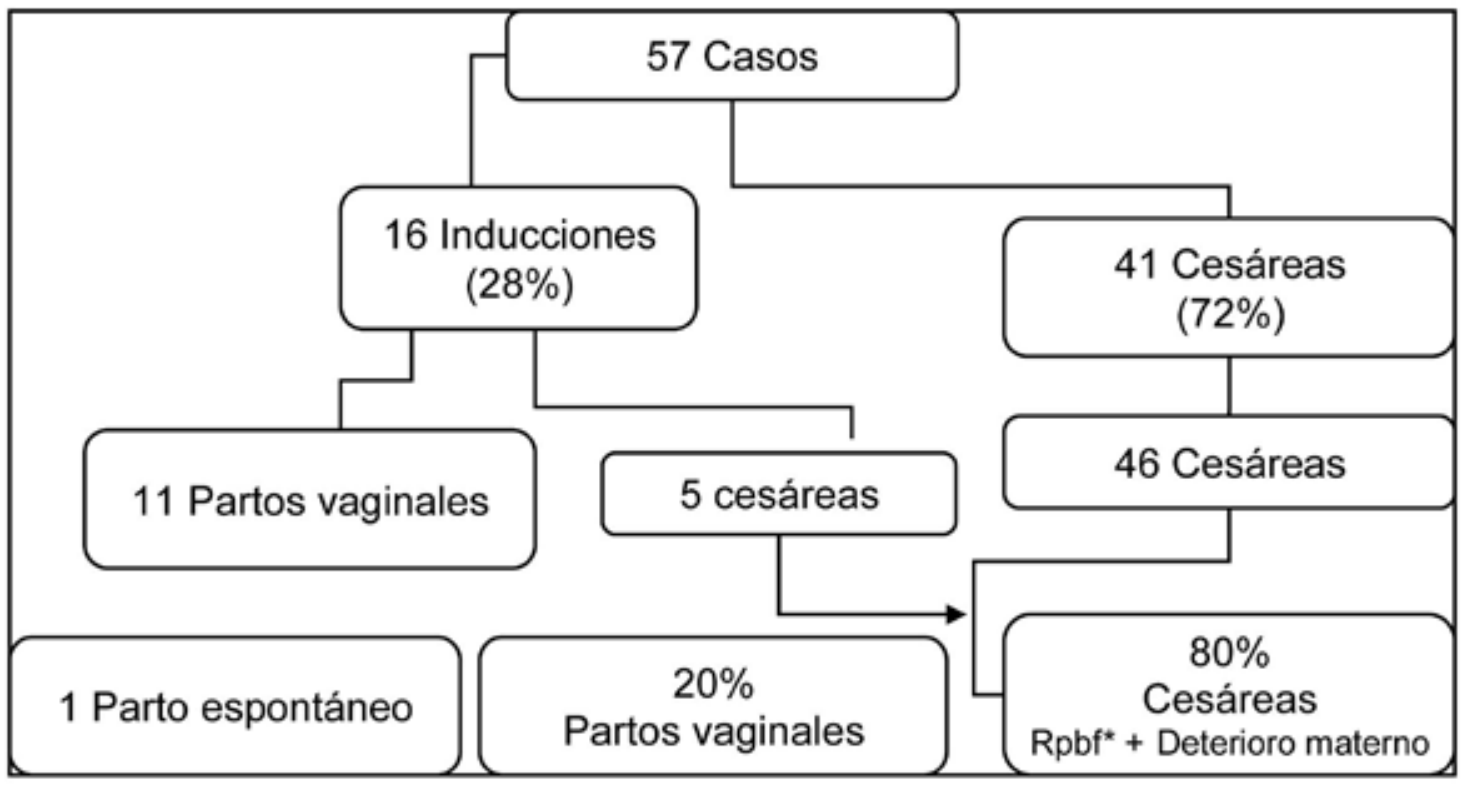

* Rpbf: Riesgo de pérdida de bienestar fetal

Figura 1. Resultados de vía de parto. 
Tabla II

MANEJOY RESULTADO PERINATAL

\begin{tabular}{cccccccccc}
\hline Paciente & EG & CIR & AUr & ACMp & Ductus & VE & PF & Apgar & pH \\
\hline 1 & $35+3$ & si & no & normal & normal & PN & 1780 & $8 / 8$ & 7.12 \\
2 & $27+5$ & si & si & si & normal & PN & 765 & $0 / 0$ & \\
3 & $33+6$ & & no & si & normal & PN & 2290 & $9 / 9$ & 7.35 \\
4 & $36+4$ & si & no & si & normal & PN & 1860 & $9 / 9$ & 7.14 \\
5 & $24+4$ & no & no & si & normal & PE & 760 & $2 / 4$ & - \\
6 & $32+2$ & si & no & si & normal & PN & 1420 & $0 / 0$ & - \\
7 & $38+3$ & si & si & si & patológico & CST & 2650 & $8 / 9$ & - \\
8 & $34+3$ & si & no & si & normal & PN & 1575 & $9 / 9$ & 7.36 \\
9 & $26+6$ & si & si & si & patológico & PN & 765 & $0 / 0$ & - \\
10 & $36+1$ & - & no & si & normal & CST & 2485 & $9 / 9$ & 7.28 \\
11 & $34+5$ & si & no & si & nomal & PFo & 2200 & $9 / 9$ & 7.25 \\
12 & $33+2$ & si & no & si & - & CST & 1300 & $7 / 8$ & 7.24 \\
13 & $35+1$ & si & no & si & - & CST & 1400 & $9 / 9$ & 7.24 \\
14 & $35+2$ & - & no & no & - & PN & - & $6 / 8 / 8$ & 7.33 \\
15 & 35 & si & no & si & normal & CST & 1590 & $9 / 9$ & 7.30 \\
16 & $35+6$ & si & no & no & normal & PN & 1560 & $9 / 9$ & 7.36 \\
\hline
\end{tabular}

PG: Prostaglandinas intravaginales; Dx: Diagnóstico; PF: Peso fetal (g); EG: Edad gestacional (semanas); CIR: Crecimiento intrauterino restringido; AUr: Arteria umbilical reversa; OliA: Oligoamnios; ACMp: Arteria cerebral media patológica (centralización); VE: Vía de extracción; CST: Cesárea segmentaria transversa; PN: Parto normal; PE: Parto espontáneo; PF: Peso fetal; PFo: Fórceps.

Tabla III

MORTALIDAD PERINATAL. ANTECEDENTES CLÍNICOS Y MANEJO

\begin{tabular}{|c|c|c|c|c|c|c|c|c|c|}
\hline Paciente & $\mathrm{AP}$ & EG & CIR & ACMp & Ductus & AUr & VE & PF & Apgar \\
\hline 1 & $\begin{array}{l}\text { HTA tras PE } \\
\text { en gestación } \\
\text { previa }\end{array}$ & $27+5$ & si & si & normal & si & PV & 765 & $0 / 0$ \\
\hline 2 & $\mathrm{PE}$ & $32+2$ & si & si & normal & no & PV & 1420 & $0 / 0$ \\
\hline 3 & $\begin{array}{l}\text { PE con } \\
22 \mathrm{~s}\end{array}$ & $26+6$ & si & si & patológico & si & PV & 765 & $0 / 0$ \\
\hline 4 & $\begin{array}{l}\text { Hemorragia } \\
\text { postparto en } \\
\text { gestación } \\
\text { previa }\end{array}$ & 30 & si & si & normal & no & CST & 855 & $9 / 9$ \\
\hline 5 & - & $29+3$ & si & si & normal & si & CST & 725 & $6 / 8$ \\
\hline 6 & $\begin{array}{l}\text { HTA previa a } \\
\text { embarazo }\end{array}$ & $27+6$ & si & & normal & no & CST & 625 & $7 / 7 / 8$ \\
\hline 7 & $\begin{array}{l}\text { Parkinson. } \\
\text { PE grave }\end{array}$ & $25+4$ & si & si & normal & no & CST & 500 & $2 / 6 / 6$ \\
\hline 8 & TRA. EHG. & $28+4$ & si & si & normal & no & CST & 640 & $6 / 7 / 9$ \\
\hline 9 & $\begin{array}{l}\text { RPM 20+4 } \\
\text { semanas }\end{array}$ & $24+4$ & no & si & normal & no & PV & 780 & $2 / 4$ \\
\hline 10 & $\begin{array}{l}\text { HTA crónica } \\
\text { CST previa por } \\
\text { PE }\end{array}$ & $28+4$ & si & si & normal & no & CST & 898 & $4 / 6 / 6$ \\
\hline
\end{tabular}

EM: Edad materna; AP: Antecedentes personales; EG: Edad gestacional (semanas); CIR: Crecimiento intrauterino restringido; PE: Preeclampsia; ACMp: Arteria cerebral media patológica (centralización); AUr: Arteria uterina reversa; VE: Vía de extracción; CST: Cesárea segmentaria transversa; PV: Parto vaginal; PF: Peso fetal (g), TRA: Terapia de reproducción asistida; EHG: Enfermedad hipertensiva gestacional. 


\section{COMPLICACIONES NEONATALES}

\begin{tabular}{|c|c|c|c|}
\hline Complicación & n (\%) & Transfusión & Intervención \\
\hline Necesidad de aminas & $14(29 \%)$ & - & - \\
\hline Alteraciones hidroelectrolíticas & $33(69 \%)$ & & \\
\hline Anemia & $24(50 \%)$ & 23 & - \\
\hline Plaquetopenia & $18(37 \%)$ & 14 & \\
\hline Ictericia & $35(73 \%)$ & - & Fototerapia: 35 \\
\hline Retinopatía & 5 & - & Fotocoagulación: 1 \\
\hline Sepsis & $\begin{array}{l}24(50 \%) \\
\text { Precoz: } 6 \\
\text { Tardía: } 18\end{array}$ & - & - \\
\hline Enterocolitis necrotizante & $4(8 \%)$ & - & Cirugía: 2 \\
\hline Membranas hialina & $24(50 \%)$ & - & Intubación: 20 \\
\hline Leucomalacia ventricular & $2(4 \%)$ & - & - \\
\hline Hemorragia intraventricular & $8(16 \%)$ & - & - \\
\hline Crisis convulsivas & $3(6 \%)$ & - & - \\
\hline Déficit neurológico tras seguimiento & $10(21 \%)$ & - & - \\
\hline Cardiopatías & $15(31 \%)$ & - & Cirugía: 2 \\
\hline Hernias & 17 (33\%) & - & Cirugía: 7 \\
\hline Audición & $\begin{array}{l}\text { Normal: } 41 \\
\text { Sin datos otras pacientes }\end{array}$ & - & - \\
\hline Rehabilitación & 12 (25\%) & - & - \\
\hline Otras* & 13 & & \\
\hline
\end{tabular}

* neumotórax, isquemia de brazo derecho, onfalitis, íleos transitorios, colestasis neonatal, chalazión recurrente, perforación intestinal, hipertensión arterial neonatal, hipotiroidismo neonatal, gastroenteritis aguda por rotavirus y citomegalovirus congénito.

La edad gestacional fue otra variable clave en cuanto a resultados perinatales en nuestro estudio. Todos, salvo un óbito fetal, ocurrieron en recién nacidos menores de 30 semanas y también fueron éstos los que obtuvieron peores puntuaciones de Apgar, mayor número de complicaciones neonatales y días de ingreso.

Existe una alta tasa de cesáreas de urgencia en los intentos de inducción de nuestras pacientes, esto coincide con otros estudios como el de Boers y cols (7), los que estudiaron la vía de parto y los resultados perinatales en fetos con crecimiento intrauterino retardado en gestaciones a término en una serie holandesa entre 2000 y 2005 . No existe consenso en la vía de parto a elegir en este tipo de pacientes, y se necesitan nuevos estudios prospectivos para determinar la mejor estrategia a seguir en estos casos, aunque algunos protocolos hospitalarios españoles ya se inclinan por cesáreas electivas en los CIR tipo III, IV y V (8).

Comparando nuestros resultados perinatales con la literatura existente, Vergani y cols (9) describieron en 2005 una tasa de resultados adversos del $41 \%$, donde se incluían las muertes perinatales, en una serie de 39 gestantes con Doppler umbilical con flujo ausente. Esta diferencia significativa respecto a nuestros resultados puede radicar en los criterios utilizados para definir los resultados adversos, mucho más estrictos en su serie que en la nuestra, en la que no se ha tenido en cuenta la gravedad del proceso. Por otro lado, Neto y cols (10) describen una mortalidad perinatal del $64,6 \%$ en una serie de 48 pacientes con Doppler umbilical con flujo ausente, asociados a enfermedad hipertensiva gestacional del $91 \%$, y la necesidad de extracción fetal en el $52 \%$ de los casos en menos de 24 horas tras el diagnóstico. Las diferencias de esta serie con nuestros resultados puede deberse a la mayor prevalencia de preeclampsia en su medio, al papel de la maduración pulmonar fetal para la disminución de los resultados perinatales adversos, y por otro lado la dotación y experiencia de los Servicios de Neonatología. 
La metodología utilizada para la búsqueda de información del estado de los recién nacidos en la actualidad a través de la revisión de historias clínicas ha limitado los datos obtenidos, ya que dependen directamente de que los facultativos de pediatría hacían constar en los informes clínicos, además de la pérdida de información sobre pacientes que fueron trasladadas a otros centros. Por otra parte, al realizarse en un periodo de seguimiento entre 1 y 6 años, podrían obviarse secuelas aún no diagnosticadas en recién nacidos de los últimos años estudiados.

El objetivo de cualquier protocolo de actuación desencadenado por una ecografía Doppler patológica es mejorar la morbilidad y mortalidad perinatal. En muchos casos, una intervención precoz innecesaria puede dar lugar a una morbilidad excesiva debido a prematuridad, sin embargo, un retraso de actuación puede provocar un recién nacido gravemente afectado o incluso un mortinato. Así, en edades gestacionales muy tempranas, la actuación médica es compleja y debe ir encaminada a maximizar la edad gestacional y minimizar la morbimortalidad neonatal y secuelas a largo plazo.

El momento óptimo para la extracción fetal debe de ser individualizado y consensuado por un equipo multidisciplinar ya que la descompensación fetal puede no ser predecible. De la misma forma, debe existir un consejo pediátrico según los resultados del Servicio de Pediatría de cada centro, ya que la experiencia y dotación de las unidades de cuidados intensivos neonatales va a determinar en gran medida la supervivencia de estos recién nacidos. Por otro lado, la participación de los padres es primordial para la toma de decisiones, teniendo que estar informados en todo momento del pronóstico de estos fetos y los resultados a largo plazo.

\section{CONCLUSIONES}

En nuestra serie, el Doppler con flujo umbilical ausente en diástole se relaciona con alto riesgo de morbimortalidad perinatal. El $65 \%$ de los niños tendrán secuelas de algún tipo en el seguimiento entre 1 a 6 años. El momento óptimo para la extracción fetal debe ser individualizado y resuelto junto con el consejo pediátrico y participación de los padres.

\section{REFERENCIAS}

1. Baschat AA, Hecher K. Fetal growth restriction due to placental disease. Sem Perinatol 2004;28:67-80.

2. Baschat AA. Pathophysiology of fetal growth restriction: implications for diagnosis and surveillance. Obstet Gynecol Surv 2004;59:617-2.

3. Ferrazzi E, Bozzo M, Rigano S, Bellotti M, Morabito A, Pardi G, et al. Temporal sequence of abnormal Doppler changes in the peripheral and central circulatory systems of the severely growth-restricted fetus. Ultrasound Obstet Gynecol 2002;19:140-6.

4. Baschat AA. Fetal growth disorders in high risk pregnancies management options. En: JamesDK, Steer PJ, Weiner CP, Gonik B (eds). Saunders Elsevier (Publ). Philadelphia, 2006; pp 240-71.

5. Thornton JG, Hornbuckle J, Vail A, Spiegelhalter DJ, Levene M. Infant wellbeing at 2 years of age in the growth restriction intervention trial (GRIT): multicentre randomised controlled trial. Lancet 2004;364:513-20.

6. Boers KE, Vijgen SMC, Bijlenga $D$, van der Post JA, Bekedam D, et al. Induction versus expectant monitoring for intrauterine growth restriction at term: randomised equivalence trial (DIGITAT). BMJ 2010;21:c7087.

7. Boers KE, van der Post JA, Mol BW, van Lith JM, Scherjon SA. Labour and neonatal outcome in small for gestational age babies delivered beyond $36+0$ weeks: a retrospective cohort study. J Pregnancy 2011;2011:293516.

8. Protocolo: Defectos del crecimiento fetal. Unidad Crecimiento Restringido y Preeclampsia. Servicio de Medicina Materno Fetal, Institut Clínic de Ginecologia, Obstetricia i Neonatologia, Hospital Clínic. Servicio de Obstetricia y Ginecología, Hospital Sant Joan de Deu. 2013-2016.

9. Vergani P, Roncaglia N, Locatelli A, Andreotti C, Crippa I, et al. Antenatal predictors of neonatal outcome in fetal growth restriction with absent end-diastolic flow in the umbilical artery. Am J Obstet Gynecol 2005;193:1213-8.

10. Martins M, Costa FH, Salani RM, Alencar CA. Fatores preditores para o óbito neonatal em gestações com diástole zero ou reversa na doppler velocimetria da artéria umbilical. Rev Bras Ginecol Obstet 2010;32:352-8. 